Mens

Revue d'histoire intellectuelle et culturelle

mens

\title{
L'« État français " sans la jeunesse : Lionel Groulx et la Révolution tranquille
}

\section{Éric Bédard}

Volume 16, numéro 2, printemps 2016

URI : https://id.erudit.org/iderudit/1041785ar

DOI : https://doi.org/10.7202/1041785ar

Aller au sommaire du numéro

Éditeur(s)

Centre de recherche en civilisation canadienne-française

ISSN

1492-8647 (imprimé)

1927-9299 (numérique)

Découvrir la revue

Citer cet article

Bédard, É. (2016). L' "État français » sans la jeunesse : Lionel Groulx et la Révolution tranquille. Mens, 16(2), 37-63. https://doi.org/10.7202/1041785ar
Résumé de l'article

Dès les années 1920, Lionel Groulx et l'équipe de L’Action française avaient espéré qu'un jour l'État du Québec travaillerait à l'émancipation économique et politique des Canadiens français. Au moment où ce programme semble prendre forme, grâce aux actions du gouvernement de Jean Lesage, le penseur du traditionalisme canadien-français se montre pourtant très critique. Les transformations culturelles en train de s'opérer le rendent en effet très pessimiste. Le regard désenchanté de Groulx ne tient pas seulement au processus de laïcisation, mais aussi à la représentation qu'il s'est toujours faite de la jeunesse. Or celle des années 1960 l'inquiète au plus haut point. 


\title{
L'« État français » sans la jeunesse : Lionel Groulx et la Révolution tranquille ${ }^{1}$
}

\author{
Éric Bédard \\ TÉLUQ - Université du Québec
}

\section{Résumé}

Dès les années 1920, Lionel Groulx et l'équipe de L'Action française avaient espéré qu'un jour l'État du Québec travaillerait à l'émancipation économique et politique des Canadiens français. Au moment où ce programme semble prendre forme, grâce aux actions du gouvernement de Jean Lesage, le penseur du traditionalisme canadien-français se montre pourtant très critique. Les transformations culturelles en train de s'opérer le rendent en effet très pessimiste. Le regard désenchanté de Groulx ne tient pas seulement au processus de laïcisation, mais aussi à la représentation qu'il s'est toujours faite de la jeunesse. Or celle des années 1960 l'inquiète au plus haut point.

\section{Abstract}

From 1920s on, Lionel Groulx and the team of L'Action française hoped that the Quebec State would someday work towards the economic and political emancipation of French Canada. However, when this project eventually began to take form, thanks in large part to actions taken by the government of Jean Lesage, the leading thinker of French Canadian

\footnotetext{
${ }^{1}$ L'auteur tient à remercier les trois évaluateurs anonymes pour leurs commentaires et leurs suggestions.
} 
traditionalism assumed a critical posture. The cultural changes that Quebec was undergoing rendered Groulx very pessimistic. His disillusionment was not only the result of rising secularism, but also of the shattering of his conceptions of youth. The youth of the 1960s worried him very much indeed.

Les nationalistes de la génération de Jean Drapeau ont conservé, tout au long de leur vie, une vive admiration pour Lionel Groulx. Au plus creux de la crise des années 1930, l'historien " national " leur avait témoigné toute sa confiance et les avait invités à construire un véritable «État français » en Amérique. Nulle surprise, donc, si, lors des obsèques de l'auteur de Directives et d'Orientations, Jean Drapeau fait partie des quelques proches qui porteront le cercueil de l'historien à son dernier repos ${ }^{2}$. Quelques semaines avant le triste événement, un matin enneigé de mars 1967, la limousine du maire de Montréal s'était arrêtée devant le 261, rue Bloomfield, à Outremont, la résidence qu'habitait le chanoine Lionel Groulx depuis 1939. Le premier magistrat de la plus importante métropole française d'Amérique était fier de lui faire visiter le grand chantier d'Expo 67, cette «Terre des Hommes » sur laquelle les yeux du monde seraient bientôt fixés. Belle occasion de montrer à son mentor, âgé de 89 ans, un accomplissement éclatant du Canada français. Francophile, le maire Drapeau voulait que Montréal, grâce à cette exposition universelle, fasse rayonner le génie français et montre le relèvement de sa patrie d'origine. De retour chez lui, Lionel Groulx aurait vanté les qualités de son disciple. Voilà un vrai chef, aurait-il dit à sa nièce, qui savait conjuguer progrès et fierté et qui tentait de concilier émancipation et enracinement ${ }^{3}$. Pouvait-il en dire autant des autres

2 Lionel Groulx s'éteint le 23 mai 1967.

3 L'anecdote est rapportée dans Susan Purcell et Brian McKenna, Jean Drapeau, traduit de l'anglais par Danielle Soucy, Montréal, Éditions Stanké, 1981, p. $167-$ 168, et confirmée par Pierre Patry, "In memoriam », L'Action nationale, vol. LVII, $\mathrm{n}^{\circ} 10$ (juin 1968), p. 968. 
artisans de la Révolution tranquille qui allaient transformer le Québec des années 1960? Pouvait-il en dire autant de la jeunesse frondeuse, issue du baby-boom, en train de faire vivre au Québec et à l'Occident une révolution culturelle sans précédent ${ }^{4}$ ?

Les rapports de Lionel Groulx à la Révolution tranquille continuent d'être discutés. Certains considèrent l'historien comme l'un de ses plus implacables critiques, d'autres en font un précurseur illustre. Le 23 mars 2015, sur le site du mouvement Tradition Québec, l'historien Jean-Claude Dupuis présentait Lionel Groulx comme le « contre-révolutionnaire tranquille " par excellence. Malgré les honneurs et les distinctions, ce penseur traditionaliste n'était " plus écouté par personne » durant les années 1960, malheureusement pour le Québec, selon Dupuis, un admirateur du chanoine. Ce dernier fait référence à la critique que Groulx a formulée sur le rapport Parent, lequel allait conduire à la « déchristianisation de la société canadiennefrançaise $e^{5}$ ". Deux ans plus tôt, à l'auditorium de la Grande Bibliothèque à Montréal, rempli à pleine capacité, Mathieu Bock-Côté, dans une conférence consacrée à la journée du 30 octobre 1995, présentait Lionel Groulx comme le précurseur du « Maître chez nous ", le grand inspirateur de l' "État français » et, surtout, comme le " père fondateur de la Révolution tranquille ${ }^{6}$ ". Qui a raison, qui a tort? Si les points de vue sont diamétralement opposés, ils comportent tous les deux une part de vérité.

Dans les pages qui vont suivre, après avoir présenté les rapports compliqués de Lionel Groulx à la Révolution tranquille, je souhaite insister sur sa perception de la jeunesse des années 1960. L'hypothèse

${ }^{4}$ Voir, à ce sujet, Jean-Marc Piotte, La révolution des mours : comment les baby-boomers ont changé le Québec, Montréal, Québec Amérique, 2016.

5 Jean-Claude Dupuis, "Le chanoine Lionel Groulx et le combat identitaire canadien-français ", 23 mars 2015, sur le site Mouvement Tradition Québec, [http:// www.tradition-quebec.ca/2015/03/le-chanoine-lionel-groulx-et-le-combat.html] (13 mai 2016).

${ }^{6}$ Mathieu Bock-Côté, « 30 octobre 1995 : le référendum sur la souveraineté : il était une fois l'indépendance ", dans Pierre Graveline (dir.), Dix journées qui ont fait le Québec, Montréal, VLB éditeur, 2013, p. 214. 
que je défends est que ses craintes et ses appréhensions au sujet des grandes transformations qui avaient cours furent directement liées à la représentation qu'il s'était toujours faite de la jeunesse, de la sienne propre, décrite dans son journal intime au tournant $\mathrm{du} \mathrm{Xx}^{\mathrm{e}}$ siècle, et de celle qui suivra. Je propose, en somme, de prolonger l'analyse pionnière qu'avait déjà proposée Sylvie Beaudreau sur la question? ${ }^{7}$. S’agissant de la jeunesse des années 1960, elle avait constaté le fossé qui s'était installé entre cette nouvelle génération et les idéaux traditionalistes de Groulx, mais sans chercher à cerner la place qu'avait occupée la " jeunesse " dans son ouvre et la mission qu'il lui avait assignée, au début de son engagement nationaliste.

\section{Ombre sur la Révolution tranquille}

Grâce aux travaux de Xavier Gélinas, de Stéphane Pigeon et de Sylvie Beaudreau, nous connaissons assez bien les rapports de Lionel Groulx à la Révolution tranquille ${ }^{8}$. Des rapports complexes, souvent troubles, mais somme toute assez noirs, comme le montrent bien ses écrits des années 1960. Essai de 161 pages lancé le 21 décembre 1964 devant un parterre de notables, dont le ministre Pierre Laporte qui lut une lettre élogieuse du premier ministre Jean Lesage ${ }^{9}$, Chemins de l'avenir allait avoir un certain retentissement, ne serait-ce que sur le plan des

7 Sylvie Beaudreau, «Déconstruire le rêve de nation : Lionel Groulx et la Révolution tranquille ", Revue d'histoire de l'Amérique française, vol. 56, n 1 (été 2002), p. 50-55.

8 Xavier Gélinas, La droite intellectuelle québécoise et la Révolution tranquille, Québec, Les Presses de l'Université Laval, 2007, p. 127-130; Stéphane Pigeon, Lionel Groulx, critique de la Révolution tranquille, mémoire de maîtrise (histoire), Montréal, Université de Montréal, 1999; Beaudreau, " Déconstruire le rêve de nation ".

9 " Mon admiration pour ce grand historien n'a fait qu’augmenter avec les années, écrit le premier ministre, et je me suis toujours réjoui de la moindre rencontre de ses idées et des miennes. Il a découvert qu'on n'est jamais aussi transcendentalement humain que lorsqu'on est complètement façonné par son milieu. On n'atteint à l'universel qu'en passant par la patrie » (Lettre de Jean Lesage, datée du 17 décembre 1964, citée dans Lionel Groulx, Mes mémoires, t. 4 : 1940-1967, Montréal, Éditions Fides, 1974, p. 300-301). 
ventes ${ }^{10}$. Cette méditation sur son époque de changements s'est en effet beaucoup plus vendue que Le Canada français missionnaire, dernier gros ouvrage d'histoire de Lionel Groulx lancé en grande pompe deux ans plus tôt, mais resté sur les tablettes. L'historien revient également sur la Révolution tranquille dans le huitième et dernier volume de ses Mémoires, vraisemblablement écrit durant les derniers mois de sa longue vie (vers 1966-1967).

Dans ces deux textes testamentaires, et crépusculaires à bien des égards, que dit-il au juste de la Révolution tranquille? En gros, rien de très positif. Le portrait est même extrêmement sombre, même si tout n'est pas complètement noir. C'est que, selon l'historien, il fallait être myope pour ne pas voir dans les grandes réformes des premières années du gouvernement Lesage une véritable " mystique de l'effort ${ }^{11}$ ", la " prise de conscience adulte d'un peuple adulte ${ }^{12}$ ". Lionel Groulx salue la " conversion » - le mot est important - de la classe politique à ses vues sur la nécessité de doter la nation canadienne-française d'un «État français » en vue d'être " Maître chez nous » sur les plans politique, économique et social. Dans Chemins de l'avenir et dans ses Mémoires, il cite sa célèbre préface à l'enquête de L'Action française et plusieurs extraits de conférences et d'entrevues accordées au fil des années, qui montrent noir sur blanc que, s'agissant de la nationalisation de l'hydroélectricité et de la réforme de l'État, les révolutionnaires tranquilles ne font que mettre en œuvre un programme conçu, pensé, rêvé, dès les années 1920, par la "vieille garde " nationaliste ${ }^{13}$. Ce programme nationaliste aurait normalement dû être mis en œuvre par l'Union nationale au milieu des années 1930; hélas, comme il l'écrit dans Chemins de l'avenir, Maurice Duplessis aurait sabordé les

${ }^{10}$ Groulx évoque le tirage du $10^{\mathrm{e}}$ mille (Mes mémoires, t. 4, p. 303). Sur la réception de cet essai, voir Gilles Chamberland, "Chemins de l'avenir » du chanoine Lionel Groulx, mémoire de maîtrise (histoire), Trois-Rivières, Université du Québec à Trois-Rivières, 1987.

11 Groulx, Mes mémoires, t. 4, p. 343.

12 Ibid., p. 337.

13 Lionel Groulx, Chemins de l'avenir, Montréal, Éditions Fides, 1964, p. 24. 
" espérances de $36^{14}$ " et instauré un " régime de la médiocrité continue $^{15}$ ". Plusieurs études d'histoire intellectuelle ont montré que, dans son esprit, "État français " n'était pas nécessairement synonyme d' "État indépendant ${ }^{16}$ ». Dans une conférence prononcée devant les Jeunesses patriotes, le 13 septembre 1936, publiée dans Directives, citée dans ses Mémoires, Groulx s'explique :

Quand nous parlons, en effet, d'État français, nous n'exigeons par-là nul bouleversement constitutionnel. Nul besoin, pour créer cet État, de changer un iota aux constitutions qui nous régissent. Nous demandons tout uniment que soit fait aujourd'hui ce que, par inintelligence ou pleutrerie, nos chefs politiques n'ont pas su faire en 1867. Au lieu d'un État qui, en tant de domaines, se donne des airs d'État neutre ou cosmopolite, nous demandons un État qui, dans le respect des droits de tous, se souvienne aussi de gouverner pour les nationaux de cette province, pour la majorité de la population qui est canadienne-française ${ }^{17}$.

En somme, s'il approuvait les réformes qui permettaient au peuple canadien-français de prendre en main son économie, il n'allait pas jusqu'à endosser la cause de l'indépendance, même si le patriotisme des jeunes indépendantistes l'a toujours ému ${ }^{18}$.

14 Ibid., p. 24.

15 Ibid., p. 23. Groulx raconte une entrevue avec Maurice Duplessis qui aurait eu lieu avant la prise du pouvoir en 1936. La discussion a beau porter sur l'histoire, le courant ne passe pas. En prenant congé de son invité, Groulx se serait fait cette réflexion : "Est-ce possible que nous ayons tant travaillé, tant peiné depuis vingt ans, pour ce désolant résultat : mettre à la tête de la province un Maurice Duplessis?» (cité dans Groulx, Mes mémoires, t. 3 : 1926-1939, Montréal, Éditions Fides, 1972, p. 315).

16 Jean-Pierre Gaboury, Le nationalisme de Lionel Groulx : aspects idéologiques, Ottawa, Les Éditions de l'Université d'Ottawa, 1970, p. 151-160; Michel Bock, "Une fausse querelle : les minorités françaises et la polémique de l'État français ", dans Robert Boily (dir.), Un héritage controversé : nouvelles lectures de Lionel Groulx, Montréal, VLB éditeur, 2005, p. 49-63.

17 Lionel Groulx, Directives, Saint-Hyacinthe, Éditions Alerte, 1959, p. 109; extrait de cette conférence, citée dans Mes mémoires, t. 4 p. 346.

18 Voir Mathieu Noël, Lionel Groulx et le réseau indépendantiste des années 1930, Montréal, VLB éditeur, 2011. 
Lui d'ordinaire si méfiant à l'égard de la gente politicienne, il affiche dans ses Mémoires une certaine sympathie pour trois chefs de file de la Révolution tranquille. Il salue la conversion de Jean Lesage qui, après avoir été un centralisateur libéral à Ottawa, allait adopter les vues nationalistes de ses compatriotes; il loue le travail de réflexion de Georges-Émile Lapalme, l'auteur de l'important programme nationaliste du Parti libéral du Québec, de « 6 à 700 pages ", rapportet-il dans ses Mémoires, une " œuvre remarquable ", selon son bon ami René Chaloult ${ }^{19}$. Même René Lévesque trouve grâce à ses yeux, celui-là même que le chroniqueur Robert Rumilly et la presse de l'Union nationale présentaient comme un cryptocommuniste durant la campagne électorale de $1960^{20}$. Dans ses Mémoires, il cite un bref échange de juin 1965 qu’il aurait eu avec Lévesque :

"Vous autres, politiciens, lui disais-je, réussirez-vous à tuer ce mouvement, ce réveil comme vous en avez tué tant d'autres? »«Jamais! m’avait répondu, d'un ton brusque, le ministre Lévesque. Qui que ce soit qui gouverne à Québec [...] se verra forcé de faire ce que nous nous efforçons de faire ${ }^{21}$. »

Dans une lettre à Jean-Marc Léger envoyée quelques semaines avant sa mort, Groulx vante le travail de ce ministre sortant « qui paraissait souhaiter à la longue de nous rendre vraiment maitre chez nous ${ }^{22}$ ". Il faut dire que Xavier Gélinas a bien montré les éléments de continuité entre le nationalisme traditionaliste d'un Lionel Groulx et le nationalisme modernisateur d'un René Lévesque, du moins du

${ }^{19}$ Groulx, Mes mémoires, t. 4, p. 338. Il s'agit évidemment du document de réflexion et de doctrine rédigé par Lapalme avant l'élection de juin 1960, publié beaucoup plus tard sous le titre de Pour une politique (VLB éditeur, 1988). À l'époque de l'écriture des Mémoires, ce manuscrit n'était connu que des dirigeants libéraux.

20 Éric Bédard, « 22 juin 1960 : l'élection de Jean Lesage : "un changement de la vie” ? ", dans Graveline (dir.), Les dix journées qui ont fait le Québec, p. 204-205. On sait que Groulx avait pris ses distances de ces courants " droitiers " plus extrêmes au milieu des années 1950 (voir Xavier Gélinas, La droite intellectuelle, p. 127-130).

${ }^{21}$ Groulx, Mes mémoires, t. 4, p. 332.

22 Une lettre datée du 17 avril 1967, citée dans Pigeon, Lionel Groulx, critique de la Révolution tranquille, p. 41. 
point de vue de la conception culturelle de la nation, de la définition parfois " ambivalente " du statut politique du Québec et du rôle consenti à l'État ${ }^{23}$. Quelques jours après le décès du chanoine, Lévesque confessait son admiration pour l'historien :

J'ai connu l'abbé Groulx avant de savoir qui il était, lorsque, petit gars, je rêvais de reconquête en lisant L'appel de la race, son roman-pamphlet du début. Plus tard, heureusement pour lui et pour nous, il écrivit bien d'autres œuvres. Comme tout le monde, j'en ai fait mon profit et, de loin, admiré cet homme qui était si passionnément attaché à ce Québec pour lequel il savait se montrer si impitoyable. [...] Ceux qui l'ont vraiment connu ont certes eu de la chance. De même que le peuple qui l'a produit et a pu si longtemps compter sur lui ${ }^{24}$.

Si Groulx se réjouit, donc, de l'édification d'un "État français ", il condamne vivement, plusieurs l'ont montré, la révolution des mentalités qui a cours durant les années 1960. Il dénonce l'arrachement à la tradition, le rejet radical du passé, les pieds de nez à la morale chrétienne. Les mots qu'il utilise dans ses Mémoires, alors qu'il approche de ses 90 ans, ne manquent pas de force. Tout "paraît s'effondrer " dans ce nouveau Québec en train d'émerger. Il se sent " aussi perdu qu'un enfant dans une forêt tropicale ${ }^{25}$ ». À ses yeux, cette secousse culturelle a tout d'un "déferlement de vagues fracassantes ", nous dirions aujourd'hui d'un tsunami. "Aucun événement dans notre histoire, pas même la Conquête anglaise ne nous aura remués, ébranlés jusqu'au fond de nos assises ${ }^{26}$ ", écrit Groulx, visiblement troublé par ce qu'il voit. Ces assises, on les devine, ce sont celles de la religion catholique romaine, celles des penseurs de

${ }^{23}$ Xavier Gélinas, " Notes sur René Lévesque et le traditionalisme canadien-français », dans Alexandre Stefanescu (dir.), René Lévesque : mythes et réalités, Montréal, VLB éditeur, 2008, p. 37-49.

${ }^{24}$ Chronique du Dimanche-Matin du 28 mai 1967, reproduite dans René Lévesque, Chroniques politiques, t. 1 : 1966-1970, Montréal, Éditions Hurtubise, 2014, p. 270-271.

25 Groulx, Mes mémoires, t. 4, p. 298.

26 Ibid. 
son Église - Bossuet, par exemple, très souvent cité dans Chemins de l'avenir -, celles d'une certaine conception de ce qu'il appelle l'« homme fondamental » qui, malgré les avancées de la technique et les progrès de la science, reste foncièrement le même; cet homme fondamental dont l'âme, cette " partie supérieure et spirituelle ", doit inspirer la vie; cet homme fondamental blessé par le péché originel et pour lequel la culture et la vie bonne sont une conquête quotidienne et difficile ${ }^{27}$. Ces assises, ce sont aussi celles d'une éducation humaniste classique, censée former des hommes d'élite, un enjeu fondamental qui l'a hanté toute sa vie. S'il a de l'estime pour les Lesage, Lapalme et Lévesque, il affiche le plus grand mépris pour Paul Gérin-Lajoie et les commissaires chargés d'enquêter sur le système éducatif. Il dénonce avec la plus grande vigueur les recommandations du rapport Parent, trop soumises à une doxa utilitariste, importée des États-Unis.

Dans Chemins de l'avenir, Groulx cible les responsables de cette révolution culturelle sans précédent. Il s'en prend aux " mauvais " maîtres, les Jean-Paul Sartre, Albert Camus, Simone de Beauvoir, Henry de Montherlant et André Gide, "le plus dangereux de tous ${ }^{28}$ ", précise-t-il, tous plus ou moins disciples de Nietzsche et de Freud, ces maîtres du soupçon qui auraient "semé le doute dans l'esprit de la jeunesse ${ }^{29}$ ", dissout par leurs réflexions acides des trésors de vérités accumulées au fil des siècles. Il s'en prend aussi aux dirigeants de l'action catholique spécialisée qui « tenaient pour si peu les valeurs morales, spirituelles incarnées dans l'âme de leur petite nation ${ }^{30}$ »; il leur reproche leur catholicisme " trop abstrait, trop irréel ${ }^{31}$ ». Il s'en prend aussi aux " excès de libéralisme ${ }^{32}$ " de Cité libre et aux émissions de Radio-Canada, cette société d'État qui diffuse une " chanson

${ }^{27}$ Groulx, Chemins de l'avenir, p. 51.

28 Ibid., p. 35.

29 Ibid., p. 36.

30 Ibid., p. 41.

31 Ibid., p. 43.

32 Ibid., p. 36. 
érotique, puante souvent de la sensualité la plus désinvolte ${ }^{33}$ ". Mais outre ces critiques attendues de la part d'un traditionaliste irrité par ce modernisme philosophique et cette transformation des mœurs, ce qui frappe lorsqu'on lit Chemins de l'avenir et ses Mémoires, c'est le portrait extrêmement sombre qu'il trace de la jeunesse. On a vraiment le sentiment que c'est le mode de vie, que ce sont les valeurs de cette jeunesse des années 1960 qui le troublent le plus et le rendent si pessimiste.

Cette jeunesse nouvelle, il la voit défiler quotidiennement devant sa résidence de la rue Bloomfield, à Outremont. De sa fenêtre, il aperçoit, en fin d'après-midi, les élèves de l'école Querbes et du collège Saint-Viateur. Le spectacle de ces adolescents "débraillé[s]" le désole. "Que j'en aurai vu de ces "collages" de garçonnets et de fillettes, empoignés par le cou et par la taille et déambulant au défi de toute pudeur ${ }^{34}$. "Cette jeunesse, il la voit aussi à la télévision, en train de danser au son " de chansonnettes de la plus entière vulgarité ". Les façons qu'ont ces jeunes de se trémousser le choquent : "Comment ne pas s'affliger de ces sauteries d'épileptiques, fol effort de fillettes de dix à douze ans, les cheveux dénoués, mêlées à des bambins du même âge et qui s'ingénient à se défaire le corps, à se tordre en tous sens ${ }^{35}$. "À plusieurs reprises, il déplore que la jeunesse s'adonne à des amusements médiocres, que «le sérieux, sinon même le tragique de la vie ${ }^{36}$ " lui soient devenus étrangers. Selon lui, cette jeunesse se comporte de manière si étrange, si sauvage même, qu'il a l'impression d'assister à la naissance d'une " race nouvelle surgie d'un tronc vermoulu ${ }^{37} »$. Cette dimension biologique n'est pas sans importance chez Groulx. Il croit vraiment que les jeunes souffrent d'un déséquilibre physiologique, produit d'un surcroît vitaminique, consé-

\footnotetext{
33 Ibid., p. 40.

${ }^{34}$ Groulx, Mes mémoires, t. 4, p. 299.

35 Ibid., p. 359.

${ }^{36}$ Groulx, Chemins de l'avenir, p. 138.

37 Ibid., p. 8.
} 
quence d'une diète "suralimenté [e] en viande, en fruits, en légumes ${ }^{38}$ "! Les jeunes auraient donc un surcroît d'énergie, mais cette énergie serait mal canalisée. Le grand défaut de cette génération nouvelle serait son manque d'humilité et de gratitude. Cette jeunesse, déplore Groulx, "n'a foi qu'en [elle]-même ${ }^{39}$ ", elle se moque du passé et n'a que faire des traditions; aucune autorité ne s'impose à elle. La philosophie éducative de ses maîtres fut celle de l'Émile de Jean-Jacques Rousseau, cette " théorie du bon sauvage ${ }^{40}$ ", complètement " dépassée » à ses yeux et contraire à la Vérité. Laissée à elle-même, laissée dans l'état de nature, cette jeunesse donne libre cours à ses plus bas instincts, s'adonne à une sexualité débridée, corrompt la jeune fille vertueuse.

Il est beaucoup question de sexualité dans Chemins de l'avenir. Ses positions sont loin de celles des personnalistes chrétiens qui, depuis les années 1930, prônent un nouveau rapport au corps et à la femme et souhaitent transmettre une vision moins austère de la sexualité $^{41}$. Les perspectives de Groulx sont celles d'un homme du $\mathrm{XIX}^{\mathrm{e}}$ siècle et du traditionalisme, celles des " orthodoxes " de l'Église catholique du Québec étudiés par Gaston Desjardins et qui voyaient dans la chasteté des adolescents une maîtrise de soi, un exercice de la volonté, une manière de se dominer ${ }^{42}$. Groulx assimile la sexualité à la bête en nous et continue de prôner l' " ascèse chrétienne ". L'homme des années 1960 aurait, selon lui, "perdu sur son corps l'empire souverain de jadis ». Il ne serait plus capable de réprimer ses " instincts ", ses " fantaisies ", ses " passions ». Esclave de ses désirs, l'homme des années 1960 ne pourrait plus se concentrer sur sa « vie

38 Ibid., p. 11.

39 Ibid., p. 15.

40 Ibid., p. 14.

${ }^{41}$ Voir Michael Gauvreau, The Catholic Origins of Quebec's Quiet Revolution, 1931-1970, Kingston, McGill-Queen's University Press, 2005, p. 77-119; Gaston Desjardins, L'amour en patience : la sexualité adolescente au Québec, 1940-1960, Montréal, Presses de l'Université du Québec, 1995.

42 Desjardins, L'amour en patience, p. 79-96. 
intérieure ». Or " point d'âme libre sans ascèse ${ }^{43}$ ". Parmi les principales " tâches sociales " fixées par Groulx à la jeunesse, il y a celle, qui ne manque pas de faire sourire aujourd'hui, de réhabiliter " la jeune fille " et celle de restaurer la famille comme institution. Il considère les naissances hors mariage comme un indice de relâchement qui résulte des fréquentations trop intimes avant le mariage ou de cette croyance illusoire au "grand amour ", auquel succomberaient trop de jeunes filles. «On souhaiterait que la morale chrétienne lâchât la bride à tous les instincts sexuels. Mais où aboutiraient la moralité d'une nation, la virilité de l'homme, le respect de la femme transformée plus que jamais en jouet des passions masculines ${ }^{44}$ ? "

\section{Former des croisés, des saints et des héros}

Pour saisir la nature de ce diagnostic extrêmement sévère, il faut remonter dans le temps. Toute sa vie, Lionel Groulx fonde d'immenses espoirs dans la jeunesse. C'est d'elle que devait surgir le renouveau, d'elle que viendrait la régénération morale de la nation, le rayonnement de l'Église catholique romaine en Amérique. Les combats de Groulx prennent forme à un moment où la jeunesse s'organise à l'université, dans les collèges et à l'intérieur de l'Église au sein de multiples associations dédiées à l'action catholique dite spécialisée ${ }^{45}$. La plupart des chercheurs qui se sont penchés sur ce surgissement de la jeunesse comme catégorie relativement autonome montrent l'importance jouée par la question nationale. Élites dirigeantes de demain, défenseurs de nouveaux types de solidarité, les jeunes canadiens-français, tout au long $\mathrm{du} \mathrm{xx}^{\mathrm{e}}$ siècle, furent également sommés de prendre fait et cause pour la survivance et l'épanouissement d'un peuple aux assises fragiles ${ }^{46}$. En 1914, rapporte

${ }^{43}$ Groulx, Chemins de l'avenir, p. 88-89.

${ }^{4}$ Ibid., p. 135.

45 Louise Bienvenue, Quand la jeunesse entre en scène: L'Action catholique avant la Révolution tranquille, Montréal, Éditions du Boréal, 2003.

46 Jean-Marie Fecteau, "Jeunes et sociétés québécoise et canadienne. 1. Les jeunes et leurs mouvements au Québec des $\mathrm{XIX}^{\mathrm{e}}$ et $\mathrm{XX}^{\mathrm{e}}$ siècles : quelques jalons pour une 
l'historienne Karine Hébert, des étudiants de l'Université Laval à Montréal lançaient une enquête sur la " jeunesse " de leur temps. Comme le montre ce texte signé par Jacques Hermil, paru dans L'Étudiant du 24 avril 1914, la question nationale était au centre de leurs préoccupations :
À l'heure où l'on s'inquiète un peu partout, dans nos milieux canadiens-français, de savoir si notre race est destinée à survivre, - tout en conservant sa langue et ses traditions, - aux âpres attaques dont elle est victime de la part de nos concitoyens anglophones et irlandais; au moment même où l'on crie partout que la jeunesse actuelle saura lutter plus tard avantageusement, et à armes égales contre nos adversaires nous avons cru qu'il était opportun d'interroger les jeunes pour savoir d'eux-mêmes, s'ils se sentaient préparés pour les «luttes de demain ", s'ils entraient avec confiance dans la vie ${ }^{47}$.

Durant les fertiles années de sa prime jeunesse, alors qu’il quitte les rives de l'adolescence et doit faire des choix très lourds de conséquences pour son avenir, Lionel Groulx tient un journal intime qui permet d'entrevoir les grandes missions qu'il assignera à la jeunesse tout au long de sa vie. Dès les premières pages de ce long texte, écrit de 1895 à 1911 - après décembre 1903, les entrées sont cependant moins nombreuses -, on est frappé par le ton très personnel. Le lecteur découvre un être d'une extrême sensibilité, qui se plaint de ne pas recevoir assez de lettres de sa famille, s'ennuie de ses proches et de son patelin, réfléchit à son destin. Le jeune qu'on découvre, surtout dans la première partie du journal, est tourmenté, fragile. Sans que les noms soient clairement mentionnés, sans qu'il explique la nature des déceptions qui l'affligent, il est beaucoup question

analyse historique", dans Commission internationale d'histoire des mouvements sociaux et des structures sociales, La jeunesse et ses mouvements : influence sur l'évolution des sociétés auX XIX et XX siècles, Paris, Éditions du Centre national de recherche scientifique, 1992, p. 322-327.

${ }^{47}$ Cité dans Karine Hébert, Impatient d'être soi-même : les étudiants montréalais, 1895-1960, Montréal, Presses de l’Université du Québec, 2008, p. 73. 
d'amitiés déçues. "Dévoré du besoin d'aimer ", note-t-il le 24 septembre $1897^{48}$, il est très conscient que l'amitié est chez lui une corde bien sensible. "C'est presque un malheur d'avoir une âme qui chante ou qui pleure ainsi à tous les vents ", écrit-il le 30 janvier $1898^{49}$. Ces passions tristes le plongent en lui-même et alimentent ce que, déjà, il considère être la grande maladie de la jeunesse : la mélancolie. Ce mal, il se plaint régulièrement d'en être la victime. «Il me semble que la mélancolie a élu sa demeure au-dedans de moimême, pour n'en plus partir, écrit-il, durant la soirée du 18 janvier 1896. Je n'ose plus croire au bonheur; non, non souffrir, toujours souffrir, c'est là mon partage ${ }^{50} ! »$

Plus le jeune Lionel Groulx écrit, plus on sent émerger une certaine dureté face à ce qui prend la forme d'échecs successifs qui minent son moral et la confiance qu'il porte aux autres. Comme s'il souhaitait se protéger émotivement, il en vient peu à peu à renoncer au monde et, pour le dire comme Pierre Trépanier, à " placer la divinité au centre de son affectivités1 $»$. Il écrit :

J'ignore si ce sont là ce qu'on est convenu d'appeler des illusions, mais mes rêves à moi ne portent pas sur les attractions du monde : je méprise le monde/ et son art, ses séductions n'ont rien pu sur mes dix-huit ans. Non malgré mes faibles ressources, mes humbles talents, quand je plonge dans l'avenir, j'aime à me voir combattant. La lutte me grise et m'entraîne. Je ne suis pas né lutteur, toutefois c'est l'état de vie qui me sourirait le plus ${ }^{52}$.

Quelques mois plus tard, Groulx se moque gentiment de ceux qui poursuivent la chimère du bonheur terrestre. «Le bonheur est en

${ }^{48}$ Lionel Groulx, Journal, 1895-1911, t. 1, édition critique par Giselle Huot et Réjean Bergeron, Montréal, Les Presses de l'Université de Montréal, 1984, p. 345.

49 Ibid., p. 367.

50 Ibid., p. 142.

51 Pierre Trépanier, "Ascèse et action : les impatiences de Lionel Groulx (18991906) ", dans Lionel Groulx, Correspondance, 1894-1967, t. 1 : Le prêtre-éducateur: 1894-1906, édition critique par Giselle Huot, Juliette Lalonde-Rémillard et Pierre Trépanier, Montréal, Éditions Fides, 1989, p. lxxxi.

52 Entrée du 30 mars 1897, dans Groulx, Journal, 1895-1911, t. 1, p. 292-293. 
haut ", note-t-il le 31 juillet 1897 . En novembre, il revient sur le même thème : "Pauvre vie humaine! Ce n'est qu'un désenchantement d'un bout à l'autre. Je voudrais pouvoir la secouer, la rejeter. Et pourtant non : je sens quand je mets la main sur mon cœur qu'il y a quelque chose qui sent le besoin de se dévouer, de se sacrifier ${ }^{53}$."

Comme le fait remarquer Pierre Trépanier, grand exégète de sa correspondance et de son ouvre, la vocation religieuse ne s'impose chez Groulx qu'à partir de 1899, alors qu'il vient tout juste de franchir le cap de la vingtaine et qu'il est forcé de choisir une " carrière ». Ce choix semble même avoir été très déchirant, à tout le moins problématique, comme le montre cette entrée du 26 mars 1901 :

Oui, j'ai connu comme vous et plus que vous peut-être cet écartèlement suprême d'une âme que le monde et que Dieu réclament à la fois. Feuille devenue le jouet des vents, j'ai traîné sur les hauteurs sereines et dans la poussière des vallons. J'ai dû goûter jusqu'à cet abattement extrême d'une âme qui se jette éplorée devant le Tabernacle de Jésus et qui se relève inconsolée avec tous ses troubles et toutes ses obscurités. $\mathrm{O}$, amis il n'y a pas d'angoisse pareille à cette angoisse ${ }^{54}$ !

C'est que les héros du très jeune Groulx ne s'appellent pas Ignace Bourget ou Louis-François Laflèche mais Daniel O'Connell et Louis Veuillot, deux laïcs qui, dans le premier cas, avait porté le destin de sa patrie irlandaise sur ses épaules et qui, dans le second, dans les pages de L'Univers, avait défendu les idéaux ultramontains d'une Église traditionaliste contre les « erreurs » d'une modernité rugissante. Tout indique que le jeune Groulx envisageait un tel destin, celui d'un journaliste catholique de combat. Mais pour s'y préparer, encore fallait-il lire, étudier, écrire, former des disciples. La voix de Jules-Paul Tardivel, qu'il admirait et dont il lisait la prose véhémente dans

53 Entrée du 5 novembre 1897, dans Ibid., p. 354.

${ }^{54}$ Lionel Groulx, Journal, 1895-1911, t. 2, édition critique par Giselle Huot et Réjean Bergeron, Montréal, Les Presses de l'Université de Montréal, 1984, p. 627. 
La Vérité, n'avait qu'une bien faible portée. Quant à l'Université Laval, elle recrutait des Français ou des clercs, mais bien peu de laïcs canadiens-français. Contrairement à un Édouard Montpetit qui allait bientôt s'imposer grâce au parrainage du premier ministre Lomer Gouin en personne, le Groulx de la jeune vingtaine n'avait ni les entrées ni les réseaux pour s'y faire une place. En somme, tout indique que c'est un peu par défaut que le jeune Groulx opte pour le sacerdoce parce que le divin lui offre une voie compensatoire qui lui permet de sublimer une quête déçue et parce que les combats qu'il souhaite livrer nécessitent des assises institutionnelles solides.

Malgré ces passages à vide, ces hauts et ces bas, il deviendra prêtre. Même si des doutes le hanteront plus tard, il s'engage avec beaucoup de volonté dans cette voie qu'il s'est tracée. Par moments, sa résolution frise l'exaltation :

La Religion et la Patrie, note-t-il le 13 septembre 1897 : tels seront les deux amours constants de ma vie. À quelque carrière que Dieu me destine, mon cœur, mon âme, ma vie est à ces deux grands noms. [...] Je serai soldat [plus loin : gladiateur antique]; ma vie sera une vie militante, je combattrai tant qu'il ne plaira pas / à Dieu de briser les faibles armes qu'il m'a données. Ma vie n'est plus à moi ; elle est à celui qui me l'a donnée; elle est ensuite à mon pays ${ }^{55}$.

Il ne sera pas seulement prêtre-éducateur mais «berger » de tout un peuple ${ }^{56}$. Pour être à la hauteur de sa mission, il s'impose une ascèse morale, spirituelle et physique impressionnante. En août 1903, il médite sur la question de la chasteté $e^{57}$. Il y voit un test concret de pureté, la preuve d'une "âme forte ». Aux yeux du jeune Groulx, la sexualité fait partie de ces mirages terrestres, elle est implicitement associée à la "boue " et au " péché " dans lesquels l'homme serait né - ces images seront celles de Chemins d'avenir, beaucoup plus tard.

55 Groulx, Journal, 1895-1911, t. 1, p. 341.

56 Marie-Pier Luneau, Lionel Groulx, le mythe du berger, Montréal, Leméac éditeur, 2003.

57 Groulx, Journal, 1895-1911, t. 2, p. 772-778. 
Il ne s'agit pas seulement de pratiquer l'abstinence, mais de chasser toutes les pensées impures de son esprit. "Il n’est point de péché où la distance soit si minime entre l'acte et le désir. Quand il s'agit d'impureté, abdiquer la moindre parcelle de la prépondérance de son âme, c'est céder tout à la bête ${ }^{58}$."

L'ascèse qu'il esquisse dans les pages de son journal et qu'il pratique, c'est celle des croisés, complètement dédiés aux causes de la Religion et de la Patrie. Il s'agit d'un idéal exigeant mais fait sur mesure pour des jeunes pleins d'énergie, constamment guettés par la mélancolie en même temps qu'attirés par de grandes causes. Ces caractères bien trempés, ces volontés en marche, ces athlètes de l'esprit seront nécessaires pour résister aux tentations du monde moderne et pour affronter des ennemis qui avancent souvent masqués, les libres penseurs, mais surtout les « Juifs, les Francs-maçons, les révolutionnaires ", cette " trinité de haine basse et féroce » à laquelle il s'en prend régulièrement dans les pages de son journal. La patrie canadienne-française, d'obédience catholique, rameau d'une France gouvernée par des anticléricaux qui avaient tourné le dos à son héritage chrétien et à sa vocation civilisatrice, était, elle aussi, menacée par ces valeurs modernes. Pour éviter cette dangereuse contamination, il fallait une jeunesse bien formée et moralement immunisée contre les tentations superficielles du monde terrestre. Adepte d'une conception organiciste des sociétés, Groulx considérait les nations et les civilisations, comme des êtres vivants qui devaient être régénérés par la jeunesse :

Le mal est profond; il est à la racine de l'arbre. Si le tronc est déjà trop vermoulu pour espérer sérieusement d'y infuser une sève nouvelle et capable d'enrayer cette destruction imminente, une nécessité qui s'impose du moins c'est de surveiller les pousses nouvelles qui apparaissent sur cette ruine; c'est d'endiguer les sucs corrompus du vieil arbre, c'est de les empêcher de s'introduire jusqu'aux canaux de ces tiges encore jeunes et intactes; c'est de faire prendre aux rejetons des racines vivaces leur permettant

${ }^{58}$ Ibid., p. 777. 
d'aller puiser la force et la vie dans une terre encore vierge de toute corruption ${ }^{59}$.

Au printemps 1902, Jules-Paul Tardivel lance l'idée d'organiser un grand congrès de la jeunesse canadienne-française. Ce projet exalte le jeune Groulx au point qu'il s'y voit prononcer un grand discours, digne de ceux qu'il prononcera plus tard, durant les années 1930. Avant même qu'une date ne soit fixée, qu'un comité organisateur n'ait prévu les objectifs du rassemblement, le jeune Groulx prépare une intervention forte et bien sentie :

Messieurs, je veux vous mettre en garde contre votre plus grand ennemi que vous ne soupçonnez peut-être pas. Quand on est jeune, on a l'enthousiasme facile, et quand on a le cœur bon et l'âme bien faite, il ne coûte guère de s'enflammer et de la plus belle ferveur pour un but qu'on nous a fait voir comme noble et élevé. Tout ce qui est grand et beau a des reflets et des splendeurs qui fascinent l'adolescence. Et il n'y a point d'efforts qu'elle ne soit prête à faire, fallût-il pousser jusqu'à l'héroïsme. Mais à la longue, les impressions changent : il y faut une trempe bien peu commune pour ne pas se dégoûter vite d'une lutte de tous les jours qui se termine par tant de défaites et par si peu de victoires. C'est contre ce dégoût que je voudrais vous protéger jusqu'au bout de votre carrière ${ }^{60}$.

Jeune enseignant inconnu, il rédige avec trois amis une longue lettre à Tardivel pour l'encourager dans son projet et pour lui offrir ses services. S'il devait y prendre part, note-t-il le 26 avril 1902, ce serait son "premier acte de vie publique ${ }^{61}$ ". Le congrès imaginé, appelé de tous ses vœux par Tardivel, se tiendra finalement du 25 au 27 juin 1903 à Montréal. Et c'est lors de ces assises qu'on lancera l'idée de

\footnotetext{
59 Ibid., p. 561.

${ }^{60}$ Ce discours imaginé est reproduit dans Ibid., p. 697-698. Ce passage de son journal sera repris textuellement dans la lettre envoyée à Jules-Paul Tardivel (voir Groulx, Correspondance, 1894-1967, t. 1, p. 196).

${ }^{61}$ Groulx, Journal, 1895-1911, t. 2, p. 715.
} 
fonder l'Action catholique de la jeunesse canadienne, l'ACJC, dans laquelle Lionel Groulx s’investira sans compter.

Lionel Groulx projetait sur la jeunesse de son temps les troubles qu'il avait lui-même ressentis au moment de devenir prêtre. Il tenait également pour acquis que les jeunes de son temps partageaient sa soif d'absolu et qu'ils n'attendaient qu'un signe pour être mobilisés. À lire son journal et le discours qu'il rêve de prononcer, on a le sentiment qu'il leur dit : je comprends votre idéalisme, mais je saisis aussi intimement les profondeurs abyssales de votre désarroi devant ce monde souillé par le péché, la corruption, les idées faciles; dans les moments de doute, je serai à vos côtés pour vous guider vers les lumières de la Vérité. Fruit d'angoisses personnelles surmontées et d'un idéalisme exalté, cet engagement auprès de la jeunesse sera constant tout au long de sa vie. Son plan d'action, très ambitieux, il le fixe dans les pages de son journal, le 31 mai 1902 : "Action immédiate par la régénération de la classe juvénile; élévation de l'idéal écolier. Pour cela, écrire, écrire, écrire, toujours; parler, parler encore, parler sans cesse ${ }^{62}$."

Il parlera beaucoup, prononcera des centaines de conférences; il écrira aussi beaucoup, énormément. Sans surprise, la jeunesse est le sujet de son premier livre, Une croisade d'adolescents, qui raconte les débuts de l'ACJC. La citation en exergue du livre en dit long sur sa vision de la jeunesse. Elle est d'un certain Lionel Montal, l'un des 22 pseudonymes répertoriés par Marie-Pier Luneau, auxquels a recouru Lionel Groulx durant sa longue carrière de polémiste ${ }^{63}$ :

Quelle que soit l'horreur des périls où nous sommes, Ne crains pas, mon pays, les jeunes vont venir!

Ils viennent... c'est leur pas, c'est la moisson des hommes

Qui dore à l'horizon, le champ de l'avenir.

62 Ibid., p. 753.

${ }^{63}$ Marie-Pier Luneau, Les Lionel Groulx : la pseudonymie comme stratégie littéraire et jeu institutionnel (1900-1966), mémoire de maîtrise (études littéraires), Sherbrooke, Université de Sherbrooke, 1996, p. 29. 
Quand du Droit, de l'Honneur la cause est menacée;

Quand la patrie en deuil penche vers le trépas,

Les jeunes, c'est l'espoir, la suprême pensée,

C'est la garde qui meurt mais qui ne se rend pas!

Le préambule de l'ACJC, que Groulx prend soin de citer dans son livre, est révélateur :

Concentrer vers un même but toutes les énergies d'une portion choisie de la jeunesse; leur communiquer cette puissance décuplée qui naît de l'union et d'une noble émulation, voilà toute la raison d'être de l'Action catholique [...]. Dieu et la Patrie, c'est notre ferme espérance, ne dédaigneront pas les services de jeunes hommes qui auront fait serment de renoncer à la vie de l'inertie et du laisser-faire pour embrasser la vie de la lutte et du travail ${ }^{64}$.

Groulx explique qu'en créant cette association au début du $\mathrm{xx}^{\mathrm{e}}$ siècle, lui et d'autres souhaitaient proposer à la jeunesse " un idéal haut et précis » et ainsi " rompre enfin avec la tradition de fainéantise et de badauderie épicurienne ${ }^{65}$ ». L'ambition : former des soldats de l'Église et de la Patrie.

L'ouvrage est aussitôt paru que Groulx projette de lancer une grande enquête, cette fois-ci, sur la jeunesse universitaire. En plus d'encadrer les adolescents des collèges, il importait également de canaliser l'énergie des étudiants vers de plus hautes aspirations. Il souhaitait mieux saisir le climat moral qui régnait à l'université, conforter son impression d'une jeunesse souffrant " d'inertie intellectuelle " et d'une "vie religieuse et morale [...] déprimée d6 $^{66}$; il voulait vérifier si les étudiants avaient une attitude trop " individualiste " - ce qu'il craignait - ou s'ils attachaient quelque importance aux questions nationales et sociales. C'est qu'en 1915,

${ }^{64}$ Cité dans Lionel Groulx, Une croisade d'adolescents, Québec, L’Action sociale, 1912, p. 33.

65 Ibid., p. xi-xii. Badauderie signifie « curiosité naïve ».

${ }^{66}$ Cité dans Pierre Trépanier, "Un projet d'enquête sur la jeunesse universitaire (1913) ", Les Cahiers des Dix, no 54 (2000), p. 152. 
alors qu'il donne ses premiers cours à l'Université Laval de Montréal, Groulx s'inquiète du " pessimisme " de la jeunesse, incapable de se résigner aux "faiseurs de toute espèce ", écrit-il à Henri Bourassa, parce qu' elle serait nostalgique d'un " état supérieur ${ }^{67}$ ». Il s' inquiète aussi des mœurs de la jeunesse universitaire, comme le montre une lettre adressée à Rodrigue Villeneuve et datée du 30 décembre 1916 :

[...] la jeunesse universitaire n’a rien changé à ses méthodes de vivre. C'est toujours la même légèreté, la même course aux mêmes frivolités et aux mêmes petites débauches. On traîne des barils de boisson, et on s'enivre en plein corridor d'université; les gardiens y ramassent des flacons vides à tout bout de champ [...]. Et l'un de ces jeunes gens, un jeune homme averti et parfaitement digne, me disait l'autre jour qu'on peut à l'Université aller faire son tour mensuel ou semi-mensuel dans les maisons de débauche, s'enivrer ou à peu près une fois toutes les quinzaines, et cependant garder encore, au milieu de ses camarades, la réputation d'un jeune homme comme il faut! [...] C'est tout de même un problème assez curieux que cette frénésie pour le plaisir et la frivolité, au sein d'une jeunesse qui en somme n'est pas endoctrinée par des professionnels de la sensualité et du scepticisme ${ }^{68}$.

Malheureusement pour lui, trop peu de jeunes hommes prendront le temps de répondre à son questionnaire, ce qui n'empêche pas le jeune abbé Groulx de tracer le portrait de l'étudiant universitaire "idéal de 1913 ». Le texte, retrouvé dans les archives par Pierre Trépanier, fait un peu plus de 60 pages et n’a jamais été publié. Cet étudiant idéal - nous pourrions dire, ce jeune idéal - disposerait d'une bonne culture générale, pétrie par les enseignements de Thomas d'Aquin, qui ferait de lui un "soldat de la vérité69 ». Ce jeune

67 Lionel Groulx à Henri Bourassa, 28 mai 1915, dans Lionel Groulx, Correspondance, 1894-1967, t. 3 : L'intellectuel et l'historien novices : 1906-1915, édition critique par Giselle Huot, Juliette Lalonde-Rémillard et Pierre Trépanier, Montréal, Éditions Fides, 2003, p. 648.

${ }^{68}$ Citée dans Pierre Trépanier, "L'étudiant idéal vers 1913 ", Les Cahiers des Dix, n 55 (2001), p. 127-128.

69 Ibid., p. 131. 
serait également un " résistant " religieux et un traditionaliste qui saurait résister au découragement, au cynisme et au soupçon généralisé. Il serait fier de sa foi, pratiquerait un catholicisme " apostolique » tourné vers la Cité, par opposition à un catholicisme " individualiste ", cantonné à la sphère privée. Au lieu d'embrasser toutes les nouveautés, dans le doute, il saurait faire confiance au passé et aux traditions héritées car «le sentiment contre lequel il importe le plus de protéger une minorité, c'est la défiance de soi-même, le dédain de son passé, le doute en son avenir qui préparent à l'assimilation par le mépris ${ }^{70} »$. Sans surprise, cet étudiant idéal serait un patriote engagé, présent dans l'arène, au service des siens, combatif.

Vingt ans plus tard, alors que le Canada français traverse une terrible crise économique, sociale et morale, cette jeunesse idéale, cette jeunesse tant espérée, Lionel Groulx a le sentiment de la voir émerger. Sans hésiter, il offre son soutien à l'association des JeuneCanada fondée en décembre 1932 qui publie, dans les pages de L'Action nationale de février 1933, son célèbre « Manifeste de la jeune génération ". L'année suivante, il offre son concours aux jeunes de Québec qui lancent la revue Vivre. Il soutient également les Jeunesses patriotes, un groupe indépendantiste fondé en 1936 :

Ce furent des jours réconfortants. Simple incident en apparence que cette effervescence de la jeunesse. Dans l'atonie générale et profonde, ces voix de jeunes résonnèrent comme la sonnerie stridente d'un réveille-matin. Disons comme un coup de clairon [...], la chiquenaude qui sortit l'opinion de sa torpeur ${ }^{71}$.

Cette « jeunesse de 1937 ", à laquelle il dédie la réédition du premier tome de Notre maître le passé, le gonfle d'espoir. Ce Groulx des années 1930 croyait que la jeunesse était enfin prête à poursuivre les grands desseins qu'il avait imaginés pour elle dès le début du siècle, alors qu'il n'était encore qu'un modeste professeur de collège. Confrontée au chômage, à la corruption du régime libéral, au pouvoir démesuré

${ }^{70}$ Cité dans Ibid., p. 139.

${ }^{71}$ Groulx, Mes mémoires, t. 3 : 1926-1939, p. 282. 
des trusts, qui avaient la mainmise sur les grandes richesses naturelles, la jeunesse des années 1930 cherchait des maîtres, un élan, un programme d'action. Plusieurs se tournaient tout naturellement vers Groulx et attendaient de lui " orientations » et « directives ». Groulx dit alors comprendre "l'inquiétude tragique » de la jeunesse ${ }^{72}$ et sa colère contre ses aînés, qui auraient abandonné les "attitudes traditionnelles ${ }^{73}$ ", refusé les grands défis nationaux. Cette jeunesse nouvelle s'attendait à une "doctrine ", à l'action et à des chefs énergiques, capables de lancer les grands chantiers de l'État français, en économie, en politique, en éducation. "Libérons-nous enfin de ce cauchemar de la survivance qui a trop longtemps accaparé et paralysé d'autres énergies ", lance Groulx, le 6 juin 1933 au Palais Montcalm ${ }^{74}$.

Deux mois après le retentissant discours du congrès de la langue française prononcé le 29 juin 1937, lors duquel il lance son fameux " Notre État français, nous l'aurons ", il propose une conférence intitulée "Préparation des jeunes à leurs tâches prochaines ». Au parterre rempli d'éducateurs venus l'écouter, il propose deux consignes: "Faites-nous des hommes » et «Faites-nous des saints ${ }^{75}$ »! Pour faire des hommes, il fallait de l'éducation physique, une culture intellectuelle, une solide formation sociale et nationale qui informerait les Canadiens français de leur "double domination " (économique et politique) ${ }^{76}$ et de la nécessité de doter leur nation d'un État "définiteur et protecteur du droit de puissance et de coordination ${ }^{77}$ ".

72 Lionel Groulx, Orientations, Montréal, Éditions du Zodiaque, 1935, p. 99.

73 Ibid., p. 100.

${ }^{74}$ Ibid., p. 115. Le titre de la conférence prononcée ce jour-là, reproduite dans Orientations, p. 93-116: "L'inquiétude de la jeunesse et l'éducation nationale ».

75 Une aspiration que l'on rencontre bien avant dans sa correspondance. Le 22 décembre 1911, il écrit à l'abbé Wilfrid Lebon, du Collège Sainte-Anne-dela-Pocatière : "Puisses-tu de tous tes fils faire des hommes, faire des chrétiens, des chevaliers dont l'armure aura toutes ses pièces, voilà, mon cher abbé, le vœu que t'adresse un pauvre confrère qui voit souvent ses rêves avorter " (Groulx, Correspondance, 1894-1967, t. 3, p. 348).

76 Groulx, Directives, p. 235.

77 Ibid., p. 241. 
Faire des hommes, donc, mais aussi des "saints ". La proposition étonne, mais Groulx y voit la "vocation normale de tout chrétien ". Et pour faire émerger ces " héros ", il fallait cultiver chez les jeunes une saine nature morale, c'est-à-dire une véritable " ascèse " que Groulx définit comme une "lutte pour le gouvernement de soimême ", de multiples rencontres "avec l'effort, le sacrifice ", un " hommage du corps et de l'âme à Dieu ${ }^{78}$ ». Seuls des hommes solides et des saints dévoués seraient en mesure de relever les énormes défis auxquels était confronté le Canada français des années 1930. Ces grands défis politiques, les vieilles générations les avaient négligés, notamment parce qu'elles avaient manqué de sens moral et qu'elles s'étaient vautrées dans les luttes partisanes. Seule une jeunesse plus vertueuse, plus déterminée, concentrée sur les grandes tâches collectives à accomplir plutôt que sur ses intérêts particuliers, pourrait procéder à un véritable redressement national. C'est du moins ce que donnait à entendre la conclusion lyrique du grand discours de 1937 :

Mais vous ne voyez donc pas, vous n'entendez pas ce qui s'en vient? Le souffle de grandeur, le voici qui commence à soulever une génération. Notre avenir nouveau, la jeunesse la plus intelligente, la plus allante, la plus décidée, le porte déjà dans ses yeux. Voilà pourquoi je suis de ceux qui espèrent. Parce qu'il y a Dieu, parce qu'il y a notre histoire, parce qu'il y a la jeunesse, j'espère. J'espère avec tous les ancêtres qui ont espéré; j'espère avec tous les espérants d'aujourd'hui; j'espère par-dessus mon temps, par-dessus tous les découragés. Qu'on le veuille ou qu'on ne le veuille pas, notre État français, nous l'aurons; nous l'aurons jeune, fort, rayonnant et beau, foyer spirituel, pôle dynamique pour toute l'Amérique française. Nous aurons aussi un pays français, un pays qui portera son âme dans son visage. Les snobs, les bonne-ententistes, les défaitistes, peuvent nous crier, tant qu'ils voudront : «Vous êtes la dernière génération de Canadiens français... » Je leur réponds, avec toute la jeunesse : « Nous sommes la génération des vivants. Vous êtes la dernière génération des morts $^{79}$ ! "

78 Ibid., p. 244-246.

79 Lionel Groulx, "L'Histoire, gardienne des traditions vivantes ", dans Directives, Montréal, Les Éditions du Zodiaque, 1937, p. 242. 
La suite est connue. L'élan souhaité n'aura pas lieu. C'est la guerre qui met fin à la crise économique. De retour au pouvoir en 1944, Maurice Duplessis ne propose aucune grande politique nationale. Si la jeunesse canadienne-française de l'Ontario continue d'être inspirée par les vues traditionalistes du maître jusqu'au début des années $1960^{80}$, celle du Québec se détourne peu à peu de lui. Durant les années 1950, les jeunes intellectuels sont partagés entre le libéralisme universaliste de Cité libre et un nationalisme nouveau qui prend forme dans les pages du Devoir ${ }^{81}$. Même les jeunes historiens qu’il a formés, influencés par les approches matérialiste et structuraliste qui dominent alors les sciences sociales, sans renier leur maitre, prennent en douce leur distance du providentialisme et du traditionalisme de Lionel Groulx. À l'Université de Montréal, les Maurice Séguin, Guy Frégault et Michel Brunet jugent en effet que l'histoire qui suit la Conquête de 1760 se caractérise par les reculs, les humiliations et l'aliénation ${ }^{82}$, une perspective que Groulx juge beaucoup trop noire ${ }^{83}$. Entre l'historien " national " et la jeunesse qui monte, un fossé se creuse, un dialogue de sourds s'installe. Durant les années 1960, Lionel Groulx, ce prêtre qui avait dix-huit ans au moment de l'élection de Wilfrid Laurier, quarante ans lors de la crise de la conscription, soixante et un ans lors du déclenchement de la Seconde Guerre mondiale, semble appartenir à une autre époque.

${ }^{80}$ Michel Bock, "L'Association de la jeunesse franco-ontarienne (1949-1972) : témoin et actrice d'une mutation idéologique ", dans Michel Bock (dir.), La jeunesse au Canada français : formation, mouvements et identité, Ottawa, Les Presses de l'Université d'Ottawa, 2007, p. 197-209.

${ }^{81}$ Michael D. Behiels, Prelude to Quebec's Quiet Revolution: Liberalism versus NeoNationalism, 1945-1960, Montréal, McGill-Queen's University Press, 1985; Guy Frégault, Lionel Groulx tel qu'en lui-même, Montréal, Leméac éditeur, 1978, p. 32-39.

82 Jean Lamarre, Le devenir de la nation québécoise selon Maurice Séguin, Guy Frégault et Michel Brunet (1944-1969), Sillery, Éditions du Septentrion, 1993.

${ }^{83}$ Voir sa lettre à François-Albert Angers de 1958, reproduite dans Éric Bédard et Julien Goyette (dir.), Parole d'historiens : anthologie des réflexions sur l'histoire au Québec, Montréal, Les Presses de l'Université de Montréal, 2006, p. 131-132. 
$\sin 20$

L'historiographie nous avait jusqu'ici permis de comprendre ce qui réjouissait et ce qui irritait Lionel Groulx dans la Révolution tranquille. D’un côté, il se réjouissait que l'on ait repris le " maître chez nous " des vieux nationalistes de L'Action française qui, dans les années 1920, réclamaient une intervention musclée de l'État en économie. Il s'agissait de contrer les effets délétères d'une seconde conquête et d'abandonner les vieux réflexes de ces partis alliés aux trusts, souvent dominés par des intérêts étrangers au peuple canadienfrançais. De l'autre, on savait qu'il rejetait la grande réforme de l'éducation, parce qu'elle tournait le dos à la grande tradition humaniste des collèges classiques, mais aussi parce que, au nom de la démocratisation et de la modernisation, elle singeait le système éducatif américain, trop axé sur des valeurs matérielles et utilitaires. Sans surprise, Groulx rejetait également le processus de sécularisation plus général à l'œuvre en Occident.

Si les rapports troubles de Lionel Groulx à la Révolution tranquille, parfois enthousiastes, souvent critiques, étaient assez connus, j'ai voulu éclairer un aspect de son lourd malaise, qui ressort clairement dans Chemins de l'avenir. Sa vision de la jeunesse des années 1960 offre une clef de lecture pour comprendre la profondeur de son désenchantement et de ses désillusions. Comme j'ai tenté de le montrer, les préoccupations que l'on retrouve dans Chemins de l'avenir sur l'importance de l'ascèse sont omniprésentes dans toute l'œuvre de Groulx. L'État français n'était pas, dans son esprit, qu'une superstructure politique; cet État devait être un phare de l'esprit en Amérique, le grand vaisseau d'une mission civilisatrice et spirituelle. Pour soutenir une telle œuvre, il fallait une jeunesse énergique et exaltée, comme l'avait été le jeune Groulx à la fin du XIx ${ }^{\mathrm{e}}$ siècle, fervent admirateur de Veuillot, convaincu que les « libres penseurs » menaçaient les traditions canadiennes-françaises.

Témoin des grandes réformes des années 1960, l’octogénaire Lionel Groulx pressent que l'État français engendré par cette Révolution tranquille se construit sans la sève régénératrice d'une jeunesse 
chrétienne et ascétique au service d'un grand Idéal. Dopée par les idées à la mode propagées par tous ces faux maîtres du soupçon, cette jeunesse n'avait plus l'esprit de sacrifice des croisés. Ce qu'il craint, donc, c'est que cet État français, dont il salue l'avènement, ait toutes les allures d'une coquille vide. Un grand corps, certes, mais irrigué par un sang vicié. Une superstructure politique impressionnante mais qui, sans l'infrastructure morale portée par une jeunesse idéaliste, risquait, tôt ou tard, de s'affaisser. 